\title{
Study on the effects of defects on the AC corona characteristics of stranded conductors
}

\author{
Kai He $\mathrm{H}^{\dagger}$, Xing-Liang Jiang, Zhen-Yu Li, \\ Zhong-Yi Yang, Yu-Yao Hu and Hong-Yu Zhou \\ Department of High Voltage and Insulation Technology, \\ Chongqing University, Chongqing, 400044, China \\ E-mail: hongkongforever@foxmail.com
}

\begin{abstract}
Defects can come into being on the surface of stranded conductors, which will affect the corona onset voltage (COV) of conductors. To unveil the regularities of defects' impacts on the COVs, a series of AC corona tests of different defective conductors were carried out in a corona cage. Relationships between photon numbers and applied voltages were obtained using an UV imager, and COVs of stranded conductors with different defects were measured through photon number fitting method. Results show that relationships between photon numbers and applied voltages meet quadratic relationships in AC corona process. Different defects have different effects on COVs, among which broken strands influence COVs mostly. As the defect degree deepens, COVs decrease gradually. When the outmost layer's strands are all broken, COV increases rather than decreasing, which results from the increase of equivalent diameter of conductors at the broken points.
\end{abstract}

Keywords: Defect; Stranded conductors; Photon number; Corona onset voltage.

\section{Introduction}

In the construction process of transmission lines, abrasions can occur on the surface of stranded conductors because of frictions or collisions between conductors. Besides, in the operation process of transmission lines, conductors can vibrate owing to wind force, which may cause displacements of aluminum strands [1]. Gradually, abrasions and displacements will develop into defects such as burrs, raised strands and even broken strands. In the high voltage transmission lines, these defects will distort the surface electric field of conductors, making it easily exceed the critical breakdown strength of air [2] and bringing adverse effects to the power grid [3].

So far, when it comes to the corona discharge of transmission lines, most scholars have focused on the effects of geometric parameters, surface conditions and atmospheric parameters on the corona discharge characteristics [4-7]. Little studies about the effects of stranded conductors' mechanical defects on the AC 
corona characteristics have been reported. Thus, a series of AC corona tests have been carried out in a corona cage to study the effects of mechanical defects on the AC corona characteristics of stranded conductors. Relationships between photon numbers and applied voltages are obtained using an UV imager, and the COVs of stranded conductors with different defects have been measured utilizing photon number fitting method. Besides, the effects of stranded conductors' defect type and defect degree on the AC corona characteristics have been researched and the influence mechanisms have been revealed.

\section{Test Equipments and Methods}

\subsection{Test equipments}

The AC corona tests have been carried out utilizing a corona cage, as shown in Fig. 1(a). The corona cage is a cylinder made from polymethyl methacrylate, whose length and diameter are $1000 \mathrm{~mm}$ and $300 \mathrm{~mm}$ respectively. According to Rui Lin [8], it can satisfy the requirements of AC corona tests. Based on [9], this study chooses LGJ-35/6 stranded conductors with a length of $500 \mathrm{~mm}$ as specimens. Besides, an UV imager is used to observe the development of corona discharge and a digital measuring device, PTU2000, is used to gauge temperature, humidity and air pressure, as shown in Fig. 1(c) and Fig. 1(d).

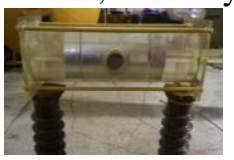

(a) Corona cage

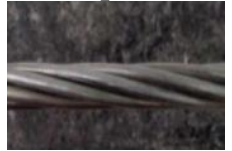

(b) LGJ-35/6

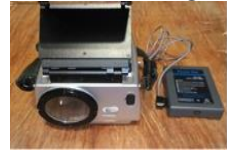

(c) UV imager

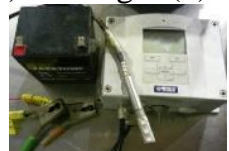

(d) PTU2000

Fig. 1. The corona cage and measuring devices

\subsection{Test methods}

(1) Before testing, defective stranded conductors with burrs, raised strands and broken strands are prepared as shown in Fig. 2.

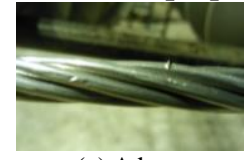

(a) A burr

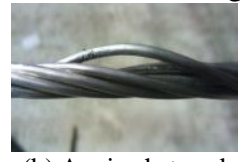

(b) A raised strand

Fig. 2. Stranded conductors with different defects

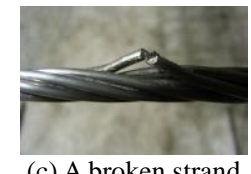

(c) A broken strand

(2) The defective stranded conductors should be placed in the corona cage. Besides, the camera lens of UV imager should aim at the defect points, and environmental parameters should be measured using PTU2000.

(3) When applying $90 \%$ of the predicted COV to the defective conductors, the boosting rate of voltage should be maintained at $0.2 \mathrm{kV} / \mathrm{s}$ until photons are 
observed through the UV imager.

(4) A thirty-second video is shot to record the photon numbers under each expected test voltage [10].

\section{Theoretical Analyses and Test Results}

\subsection{The relationship between photon number and applied voltage}

Mechanical defects can change the surface shape of stranded conductors and distort the surface electric field when the defective stranded conductors are placed in the corona cage and applied with voltages. With the increase of applied voltages, the electric field strength at defective points gets much stronger. When the ionization coefficient $\alpha$ exceeds the attachment coefficient $\eta$ [11], the initial electron avalanche will occur at defective points.

As the initial electron avalanche develops towards the outer electrode, air can be motivated and subsequently radiate photons. These photons will induce photoionization of air in the adjacent space and more photoelectrons will be generated to develop into the secondary electron emission avalanche [12]. If the total number of electrons generated by the secondary electron emission avalanche is greater than that generated by the initial electron avalanche, the corona discharge can be self-maintaining.

When the stranded conductors are placed in the center of the corona cage, there forms a typical coaxial electrodes. The corona discharge region in coaxial electrodes can be divided into two parts: the first part is the ionization region near the conductor and the second one is the electron migration region near the grounding electrode. Townsend has studied the electron distribution of the electron migration region in corona cage and obtained the relationship between corona current and DC applied voltage [13]:

$$
I=\frac{8 \pi \varepsilon_{0} \mu}{R^{2} \ln \left(R / r_{0}\right)}\left(U_{\text {app }}-U_{\text {inc }}\right) U_{\text {app }}
$$

where $U_{\text {app }}$ is the applied voltage, $U_{\text {inc }}$ is the COV, $I$ is the corona current, $\mu$ is the electron mobility, $\varepsilon_{0}$ is the dielectric coefficient of air, $R$ is the radius of external electrode, and $r_{0}$ is the radius of conductor.

In that all of the electrons in electron migration region are from ionization region, the number of electrons in migration region can reflect the corona intensity in ionization region. Suppose that $Q$ is the number of electrons in migration region and $N$ is number of photons generated in corona region per unit time, then $N$ should be proportional to $d Q / d t$. 
If $N=k \frac{d Q}{d t}=k I$, then

$$
N=\frac{8 \pi \varepsilon_{0} \mu k}{R^{2} \ln \left(R / r_{0}\right)}\left(U_{a p p}-U_{i n c}\right) U_{a p p}
$$

To simplify the form, let $A=\frac{8 \pi \varepsilon_{0} \mu k}{R^{2} \ln \left(R / r_{0}\right)}$, then

$$
N=A\left(U_{a p p}-U_{i n c}\right) U_{a p p}
$$

Eq. (3) shows a quadratic relation between $U_{\text {app }}$ and $N$, which is appropriate for DC corona. However, it has to be verified through tests that whether it is appropriate for AC corona.

\subsection{Test results and analyses}

LGJ-35/6 stranded conductors with burrs, raised strands and broken strands have been tested in the environment where the atmospheric pressure, temperature and relative air humidity are $98.41 \mathrm{kPa}, 22{ }^{\circ} \mathrm{C}$ and $60 \%$ respectively. Fig. 3 shows the relationships between photon numbers and applied voltages of different defective stranded conductors.

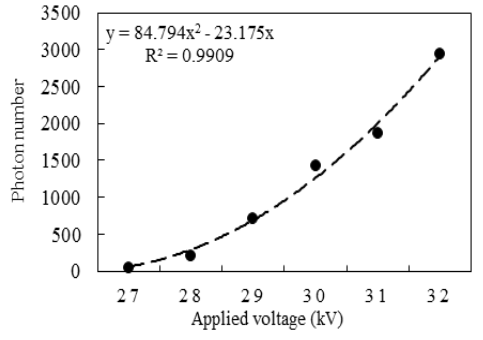

(a) Undamaged stranded conductor

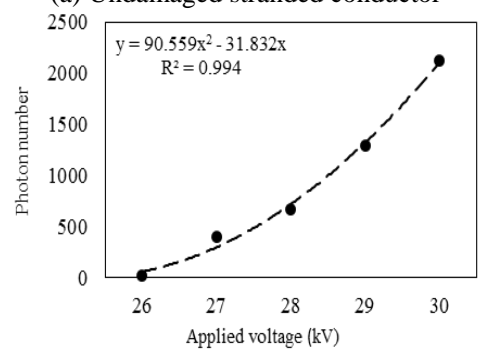

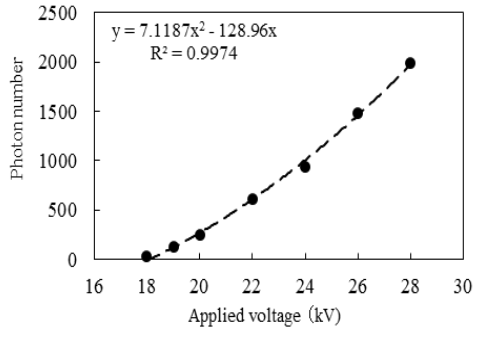

(b) Stranded conductor with a burr

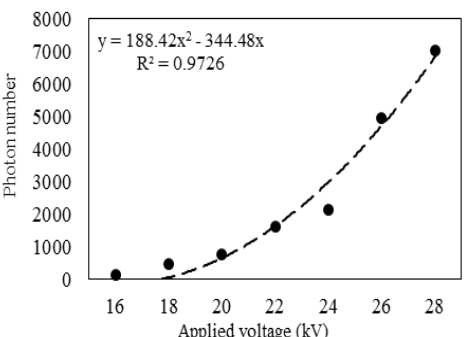

(c) Stranded conductor with a raised strand $\quad$ (d) Stranded conductor with a broken strand Fig. 3. Curve figure of photon number vs. applied voltage

Through quadratically fitting, fitting curves can be plotted with imaginary 
lines and fitting degrees can be calculated, as shown in Fig. 3. It is known that fitting degrees under different defects are more than 0.97 . Hence, a conclusion can be drawn that, relationships between photon numbers and applied voltages in AC corona process also meet quadratic relationships.

\subsubsection{The effects of defect types}

The COV of stranded conductors is an important parameter of corona characteristics, which is vital when designing EHV and UHV transmission lines. This study utilizes photon number fitting method [10] to determine COVs of different defective stranded conductors, which is briefly described as follows:

Eq. (3) can be identically transformed into:

$$
N / U_{a p p}=A\left(U_{a p p}-U_{i n c}\right)
$$

Through linearly fitting the values of $N / U_{\text {app }}$ and $U_{\text {app }}, U_{\text {inc }}$ can be obtained, which is the value of cross point where fitting line intersects with abscissa axis. Using MATLAB, different COVs can be calculated as shown in Table 1.

Table 1. Corona onset voltages of LGJ-35/6 stranded wire with different defects

\begin{tabular}{lllll}
\hline Types of defects & Undamaged & A raised strand & A burr & A broken strand \\
\hline COV $(\mathrm{kV})$ & 27.35 & 26.18 & 18.06 & 17.07 \\
$\begin{array}{l}\text { Voltage drop percentage } \\
\left|U_{i}-U_{0}\right| / U_{0} \times 100 \%\end{array}$ & 0 & $4.28 \%$ & $33.97 \%$ & $37.59 \%$ \\
\hline
\end{tabular}

From Table 1, it is known that COVs of different defects differ from each other greatly. Compared with undamaged conductor, the COV of conductor with one raised strand decreases by $1.17 \mathrm{kV}$. By contrast, the COV of conductor with one burr decreases sharply by $8.12 \mathrm{kV}$ compared with that of conductor with one raised strand. This can be explained that, different defects have different impacts on the distortion of surface electric field.

Firstly, there is no spiny part on the surface of conductor with one raised strand, and the surface electric field distribution inhomogeneity is aggravated slightly by the displacement of aluminum strands. However, the sharp part on the stranded conductor with a burr can distort the surface electric field greatly, which results in the corona onset under a relatively low applied voltage.

In addition, the COV of stranded conductor with one broken strand decreases by $0.99 \mathrm{kV}$ compared with that of the stranded conductor with a burr. It is reasonable that broken strands are the combination of raised strands and burrs: when broken strands occur, burrs can come into being at the fracture points and the aluminum strands can loose and shift easily, which can distort the 
surface electric field much more greatly. These leads to the result that the COV value of stranded conductor with one broken strand is less than that of the stranded conductor with a burr.

\subsubsection{The effects of defect degree}

LGJ-35/6 stranded conductors with 1, 2, 3, 4, 5 and 6 broken strands have also been tested in the environment where the atmospheric pressure, temperature and relative air humidity are $97.52 \mathrm{kPa}, 23.2{ }^{\circ} \mathrm{C}$ and $72.8 \%$ respectively. The COVs are shown in Fig. 4.

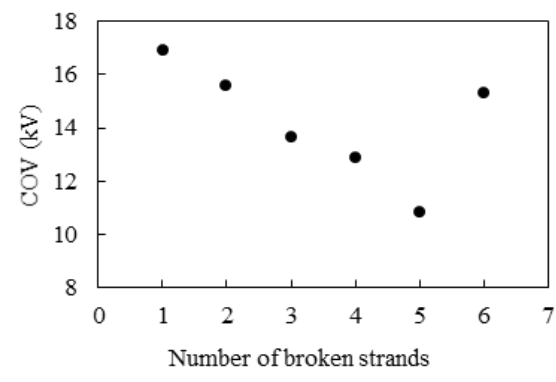

Fig. 4. Corona onset voltages of conductors with different broken strands

As shown in Fig. 4, the COVs decrease obviously with the increase of broken strands. This is because that, with the increase of broken strands, the distortion degree of surface electric field deepens severely, diminishing the applied voltage needed for corona onset. However, it has to be pointed out that, when all of the aluminum strands are broken in the outmost layer (the broken strands are connected electrically through the inner steel wire), the COV increase rather than decreasing. It is reasonable that, when all of the strands are broken, the equivalent diameter of conductors at the broken points can increase because of the displacements of strands, which can weaken the distortion of electric field and improve COVs.

\section{Conclusion}

In the $\mathrm{AC}$ corona process, relationships between photon numbers and applied voltages still meet quadratic relationships. Different defects have different effects on COVs. For burrs, raised strands and broken strands, broken strands influence COVs mostly because broken strands are the combinations of burrs and raised strands, which deepen the distortion degree of surface electric field more severely. With the deepening of defect degree, COVs decrease gradually. When the outmost layer's strands are all broken, the COV increases rather than 
decreasing, which can be ascribed to the increase of equivalent diameter of conductors at the broken points.

\section{References}

1. F. Yanting, Cause Analysis and Treatment on $500 \mathrm{kV}$ Conductor Stranded Wires Broken, Hebei Electric Power, 2014, 33, (2), pp. 4-6.

2. F. Zhiguo, Corona discharge analysis on high voltage transmission line with burrs, Heilongjiang Electric Power, 2009, 31, (6), pp. 441-443.

3. M. Abdelsalam, Monopolar corona on bundle conductors, IEEE Trans. on Power Apparatus and Systems, 1982, 101, (10), pp. 4079-4089.

4. M. Pfeiffer, Impact of Conductor Surface Type and Rain Intensity on HVDC Corona Losses, IEEE Trans. on Power Deli., 2015, 30, (5), pp. 2284-2292.

5. Q. Hu, Influence of air pressure and humidity on positive direct current corona discharge performances of the conductor in a corona cage, Int. Trans. on Electrical Energy Systems, 2014, 24, (5), pp. 723-735.

6. X. Jiang, Study on the Effects of Glaze Icing on the Corona Onset Characteristics of Stranded Conductors, IEEE Trans. on Dielectrics and Electrical Insulation, 2014, 21, (2), pp. 704-712.

7. X. Bian, The Effect of Surface Roughness on Corona-generated Electromagnetic Interference for Long-term Operating Conductors, IEEE Trans. on Dielectrics and Electrical Insulation, 2015, 22, (2), pp. 879-887.

8. L. Rui, Research on DC Positive Corona Discharge Characteristics of Conductors and Its Affecting Factors, Chongqing University, 2009.

9. Y. Shaohua, Discussion on problems of single conductor small corona cage's design, Int. Conf. on Electrical and Control Engineering, Yichang, China, 2011, pp. 2187-2190.

10. W. Zhou, A Criterion for UV Detection of AC Corona Inception in a Rod-plane Air Gap, IEEE Trans. on Dielectrics and Electrical Insulation, 2011, 18, (1), pp. 232-237.

11. M. Xu, Modified Peek Formula for Calculating Positive DC Corona Inception Electric Field under Variable Humidity, IEEE Trans. on Dielectrics and Electrical Insulation, 2012, 19, (4), pp.1377-1382.

12. X. Jiang, DC positive corona inception performances of stranded conductors and its affecting factors, Proceedings of the CSEE, 2009, 29, (34), pp. 108-114.

13. A. Robledo Martinez, Characteristics of DC corona discharge in humid reduced-density air, Journal of Electrostatics, 1993, 29, (2), pp. 101-111. 